\title{
Desempenho de substratos no cultivo do tomateiro do grupo cereja
}

\author{
Carolina Fernandes; José Eduardo Corá; Leila T Braz
}

UNESP/FCAV, Via de acesso Prof. Paulo Donato Castellane, s/n, 14884-900 Jaboticabal-SP; E-mail: carol@fcav.unesp.br; cora@fcav.unesp.br; leilatb@fcav.unesp.br

\begin{abstract}
RESUMO
Avaliou-se o desempenho de substratos compostos por areia, bagaço de cana-de-açúcar e casca de amendoim no cultivo do tomateiro do grupo cereja. O delineamento experimental adotado foi em blocos casualizados, com sete substratos e quatro repetições. Os substratos resultaram da combinação de proporções volumétricas de areia (A), bagaço de cana-de-açúcar (BC) e casca de amendoim (CA): $\mathrm{S}_{1}=\mathrm{A} ; \mathrm{S}_{2}=2 / 3 \mathrm{~A}+1 / 3 \mathrm{BC} ; \mathrm{S}_{3}=2 / 3 \mathrm{~A}+1 / 3 \mathrm{CA} ; \mathrm{S}_{4}=2 / 3 \mathrm{~A}$ $+1 / 6 \mathrm{BC}+1 / 6 \mathrm{CA} ; \mathrm{S}_{5}=1 / 2 \mathrm{~A}+1 / 2 \mathrm{BC} ; \mathrm{S}_{6}=1 / 2 \mathrm{~A}+1 / 2 \mathrm{CA} \mathrm{S} \mathrm{S}_{7}=$ $1 / 3 \mathrm{~A}+1 / 3 \mathrm{BC}+1 / 3 \mathrm{CA}$. As variáveis avaliadas foram submetidas à análise de variância e as médias comparadas pelo teste de Tukey a $5 \%$ de probabilidade. Os valores de densidade (D), espaço de aeração (EA) e água facilmente disponível (AFD) dos substratos foram estatisticamente diferentes. Os substratos apresentaram valores de $\mathrm{D}$ entre 790 e $1604 \mathrm{~kg} \mathrm{~m}^{-3}$, EA entre 2 e $21 \%$ e AFD entre 14 e $25 \%$. A produtividade do tomateiro cultivado nos diferentes substratos foi estatisticamente igual, variando entre 8,5 e $10,7 \mathrm{~kg} \mathrm{~m}^{-2}$. O bagaço de cana-de-açúcar e a casca de amendoim podem ser utilizados na composição de substratos à base de areia, para o cultivo do tomateiro do grupo cereja, cultivar Sindy, em casa de vegetação.
\end{abstract}

Palavras-chave: Lycopersicon esculentum, casa de vegetação, cultivo sem solo, propriedades físicas, fertirrigação.

\begin{abstract}
Performance of substrates on cherry tomato gowth

Cherry tomato growth was evaluated using substrates composed of sand, crushed sugar cane and peanut bark, in Jaboticabal, São Paulo State, Brazil. The experimental design was the complete randomized block with seven substrates and four replications. The seven substrates were made up of different combinations of sand (S), crushed sugar cane (CSC) and peanut bark (PB): $\mathrm{S}_{1}=\mathrm{S} ; \mathrm{S}_{2}=2 /$ $3 \mathrm{~S}+1 / 3 \mathrm{CSC} ; \mathrm{S}_{3}=2 / 3 \mathrm{~S}+1 / 3 \mathrm{~PB} ; \mathrm{S}_{4}=2 / 3 \mathrm{~S}+1 / 6 \mathrm{CSC}+1 / 6 \mathrm{~PB}$; $\mathrm{S}_{5}=1 / 2 \mathrm{~S}+1 / 2 \mathrm{CSC} ; \mathrm{S}_{6}=1 / 2 \mathrm{~S}+1 / 2 \mathrm{~PB} \mathrm{e} \mathrm{S}_{7}=1 / 3 \mathrm{~S}+1 / 3 \mathrm{CSC}+$ $1 / 3 \mathrm{~PB}$. The evaluated parameters were submitted to a variance analysis and averages were compared by the Tukey test, at 5\% probability level. The bulk density (BD), aeration space (AS) and easily available water (EAW) values were different. Bulk density ranged from 790 to $1604 \mathrm{~kg} \mathrm{~m}^{-3}$; AS, from 2 to $21 \%$; and EAW, from 14 to $25 \%$. No difference was observed among the cherry tomato yield obtained from the different substrates, which varied from 8.5 to $10.7 \mathrm{~kg} \mathrm{~m}^{-2}$. Both crushed sugar cane and peanut bark can be used to compose the substrates based on sand for the cherry tomato growth.
\end{abstract}

Keywords: Lycopersicon esculentum, greenhouse, soilless cultivation, physical properties, fertigation.

\section{(Recebido para publicação em 20 de dezembro de 2004; aceito em 24 de outubro de 2005)}

A crescente demanda por hortaliças de qualidade tem impulsionado alterações nas técnicas de produção. Verifica-se gradual substituição do cultivo de hortaliças em solo para o cultivo em substrato, principalmente quando a presença de patógenos no solo impossibilita o cultivo.

Mais do que exercer a função de suporte às plantas, o substrato para cultivo deve proporcionar adequado suprimento de ar e água ao sistema radicular. Adicionalmente, deve ser isento de fitopatógenos, de fácil manejo, baixo custo, alta disponibilidade e ter longa durabilidade.

A avaliação da qualidade de um substrato baseia-se na sua caracterização física e química. As propriedades físicas são de grande importância, uma vez que depois de acondicionar o substrato no recipiente, torna-se bastante difícil alterá-las (Verdonck, 1983).

Dentre as propriedades físicas, destacam-se a densidade, o espaço de aeração e a capacidade de retenção de água do substrato. A densidade de um substrato é definida como a massa de material sólido por unidade de volume do substrato, isto é, incluindo o espaço poroso entre as partículas (DeBoodt et al., 1974). A densidade é uma importante propriedade para o manejo, uma vez que substrato e recipiente são transportados e manipulados, devendo seu peso ser levado em conta. A densidade do substrato também influencia nos custos de transporte, manipulação e infraestrutura necessária para sua utilização.

O espaço de aeração de um substrato é definido como a proporção do volume que contém ar depois que o substrato foi saturado com água e deixado drenar a $1 \mathrm{kPa}$ de tensão (DeBoodt \& Verdonck, 1972). A capacidade de retenção de água se divide entre água facilmente disponível (volume de água liberado entre 1 e $5 \mathrm{kPa}$ de tensão), considerada acessível à planta; água tamponante (volume de água liberado entre 5 e $10 \mathrm{kPa}$ de ten- são), considerada não tão facilmente acessível à planta, e água remanescente (volume de água que permanece no substrato após aplicar a tensão de 10 $\mathrm{kPa})$, considerada não acessível para a planta (DeBoodt \& Verdonck, 1972). Entretanto, em relação ao cultivo em substratos, o que mais interessa é o espaço de aeração e o conteúdo de água facilmente disponível às plantas e não o conteúdo relativo à capacidade de retenção de água (Abad et al., 2004).

Vários são os materiais utilizados como substratos: areia, espuma fenólica, argila expandida, vermiculita, composto de lixo urbano, bagaço de cana-deaçúcar, casca de amendoim, casca de arroz, casca de pínus, fibra da casca de coco, serragem, entre outros.

A utilização de areia como substrato de cultivo para hortaliças tem apresentado resultados positivos, pois, por ter baixíssima capacidade de troca iônica, considera-se a areia um substrato de fácil manejo (Burés, 1997; Abad et al., 
2004). A maior limitação para utilização da areia como substrato é a dificuldade de manipulação devido ao peso excessivo, especialmente quando úmida (Andriolo, 1996). Apesar da alta disponibilidade de areia, atualmente, é prudente considerar que poderão ocorrer problemas em seu fornecimento no futuro, devido ao impacto ambiental causado pela sua extração (Abad et al., 2004), demonstrando a necessidade de buscar materiais alternativos para substituí-la parcial ou totalmente no cultivo de hortaliças.

Considerando-se a disponibilidade e o baixo custo, tem sido investigada a possibilidade de utilizar, como componentes de substratos, os resíduos agrícolas produzidos em cada região. Assim, observa-se que vários autores desenvolveram pesquisas com os materiais predominantes em suas regiões como a casca de arroz (Kämpf \& Jung, 1991; Andriolo et al., 1999), bagaço de canade-açúcar (Biasi et al., 1995; Fernandes et al., 2002), composto de resíduos hortícolas (Urrestarazu et al., 2000), composto de resíduo de uva (Reis et al., 2001), casca de amendoim (Fernandes et al., 2002), fibra da casca de coco (Noguera et al., 1997; Carrijo et al., 2004).

Devido às atividades agrícolas, bagaço de cana-de-açúcar e casca de amendoim são resíduos disponíveis na região de Jaboticabal, podendo, portanto, serem utilizados na composição de substratos. O objetivo deste trabalho foi avaliar o desempenho de substratos compostos por areia, bagaço de canade-açúcar e casca de amendoim no cultivo do tomateiro do grupo cereja.

\section{MATERIAL E MÉTODOS}

$\mathrm{O}$ experimento foi conduzido em casa de vegetação na Faculdade de Ciências Agrárias e Veterinárias da UNESP em Jaboticabal. A altitude local é de 613,68 m, com latitude de $21^{\circ} 14^{\prime} 05^{\prime \prime} \mathrm{S}$ e longitude de $48^{\circ} 17^{\prime} 09^{\prime \prime}$ W. O clima é do tipo Aw com transição para Cwa. A casa de vegetação foi construída em estrutura metálica, do tipo teto em arco, com $3 \mathrm{~m}$ de pé-direito, $30 \mathrm{~m}$ de comprimento e $8 \mathrm{~m}$ de largura, coberta com filme de polietileno trans- parente aditivado contra raios ultravioleta com 150 micrômetros de espessura e as laterais protegidas com telas de polipropileno preto com $50 \%$ de sombreamento. A temperatura e a umidade relativa do ar no interior da casa de vegetação foram obtidas por meio de um termohigrógrafo com registro contínuo dos valores. $\mathrm{O}$ aparelho foi instalado em abrigo de madeira a 1,0 m de altura no centro da casa de vegetação. Durante o ciclo da cultura, as médias mensais para a temperatura máxima diária nos meses de fevereiro, março, abril e maio foram, respectivamente, $39 ; 36 ; 33$ e $29^{\circ} \mathrm{C}$; para a temperatura mínima diária $21 ; 18 ; 15$ e $11^{\circ} \mathrm{C}$; para a umidade relativa do ar máxima diária 93; 95 ; 97 e $99 \%$ e para a umidade relativa do ar mínima diária 37; 38; 39 e $40 \%$.

O delineamento experimental adotado foi em blocos casualizados, com sete substratos e quatro repetições. A unidade experimental foi representada por quatro vasos, contendo uma planta por vaso. Os vasos utilizados foram de plástico marrom, com capacidade para $5 \mathrm{~L}$, com $25 \mathrm{~cm}$ de diâmetro de boca, 17 $\mathrm{cm}$ de diâmetro de fundo, $18 \mathrm{~cm}$ de altura e 8 furos na base.

Os substratos resultaram da combinação de proporções volumétricas de areia (A), bagaço de cana-de-açúcar (BC) e casca de amendoim (CA). A areia utilizada foi a conhecida comercialmente como areia média. $\mathrm{O}$ bagaço utilizado foi o resíduo fibroso resultante da extração do caldo dos colmos da canade-açúcar. A casca de amendoim utilizada foi moída e passada em peneira com abertura de $6 \times 18 \mathrm{~mm}$. As combinações obtidas foram: $S_{1}=A ; S_{2}=2 / 3$ $\mathrm{A}+1 / 3 \mathrm{BC} ; \mathrm{S}_{3}=2 / 3 \mathrm{~A}+1 / 3 \mathrm{CA} ; \mathrm{S}_{4}=2 /$ $3 \mathrm{~A}+1 / 6 \mathrm{BC}+1 / 6 \mathrm{CA} ; \mathrm{S}_{5}=1 / 2 \mathrm{~A}+1 /$ $2 \mathrm{BC} ; \mathrm{S}_{6}=1 / 2 \mathrm{~A}+1 / 2 \mathrm{CA}$ e $\mathrm{S}_{7}=1 / 3 \mathrm{~A}$ $+1 / 3 \mathrm{BC}+1 / 3 \mathrm{CA}$.

Cada componente dos substratos foi caracterizado física e quimicamente. As propriedades físicas avaliadas foram densidade (D), segundo Hoffmann (1970), descrito por Backes (1988); distribuição do tamanho de partículas (granulometria), utilizando peneiras de 4,$0 ; 2,0 ; 1,0 ; 0,5 ; 0,25$ e $0,125 \mathrm{~mm}$ de abertura, e espaço de aeração (EA) e água facilmente disponível (AFD), se- gundo DeBoodt \& Verdonck (1972). As propriedades químicas avaliadas foram: pH e condutividade elétrica (CE), segundo método de extração por volume na proporção de 1:1,5 (substrato:água) (Sonneveld et al., 1974). Os valores das propriedades avaliadas na caracterização dos componentes foram: $\mathrm{D}=1691$ $\mathrm{kg} \mathrm{m}^{-3}, \mathrm{EA}=2 \%, \mathrm{AFD}=23 \%, \mathrm{pH}=5,3$ e $\mathrm{CE}=0,02 \mathrm{dS} \mathrm{m}^{-1}$ para a areia; $\mathrm{D}=58$ $\mathrm{kg} \mathrm{m}^{-3}, \mathrm{EA}=27 \%, \mathrm{AFD}=12 \%, \mathrm{pH}=$ 5,1 e $\mathrm{CE}=0,08 \mathrm{dS} \mathrm{m}^{-1}$ para o bagaço de cana-de-açúcar; $\mathrm{D}=186 \mathrm{~kg} \mathrm{~m}^{-3}, \mathrm{EA}=$ $24 \%, \mathrm{AFD}=14 \%, \mathrm{pH}=5,4$ e CE $=0,82$ $\mathrm{dS} \mathrm{m}^{-1}$ para a casca de amendoim. A distribuição do tamanho de partículas encontra-se na Tabela 1.

Posteriormente, os componentes foram misturados nas combinações e a granulometria dos substratos (Tabela 1) foi determinada como descrito anteriormente. Procedeu-se então, o preenchimento dos vasos com os substratos. No centro de cada vaso, foi colocado um anel volumétrico de PVC de $285 \mathrm{~cm}^{3}$ (7,2 cm de diâmetro e 7,0 cm de altura), com o objetivo de caracterizar as condições físicas dos substratos no final do cultivo. Para tanto, após o cultivo, os vasos foram desmontados e os anéis retirados cuidadosamente para a determinação de D, EA e AFD.

As mudas do tomateiro cultivar Sindy, híbrido $\mathrm{F}_{1}$ do tipo cereja, foram transplantadas no estádio de quatro folhas definitivas. Conduziu-se uma planta por vaso com duas hastes por planta no espaçamento de 1,0 x 0,5 m (2 plantas $\mathrm{m}^{-2}$ ). A desbrota e a condução vertical da cultura foram realizadas semanalmente. A poda dos ponteiros foi realizada quando as plantas atingiram dois metros de altura.

$\mathrm{O}$ volume de água aplicado por irrigação foi o necessário para promover a drenagem dos vasos preenchidos com o substrato areia, de acordo com o critério visual. $\mathrm{O}$ volume de água drenado não foi medido, mas foi o mínimo necessário para perceber a drenagem dos vasos. A mínima drenagem dos vasos foi utilizada para garantir a uniformidade na distribuição de água no substrato acondicionado no vaso. Durante o cultivo, o volume de água aplicado por irrigação aumentou de acordo com o estádio de desenvolvimento da cultura. $\mathrm{O}$ 
Tabela 1. Distribuição do tamanho de partículas dos componentes e dos substratos utilizados no cultivo do tomateiro do grupo cereja, cultivar Sindy, em casa de vegetação. Jaboticabal, FCAV-UNESP, 2004.

\begin{tabular}{lrrrrrrr}
\hline & \multicolumn{7}{c}{ Diâmetro das partículas (mm) } \\
\cline { 2 - 8 } Componentes/Substratos & $\mathbf{0}, \mathbf{1 2 5}$ & $\mathbf{0 , 1 2 5 - 0 , 2 5}$ & $\mathbf{0 , 2 5 - 0 , 5}$ & $\mathbf{0 , 5 - 1}$ & $\mathbf{1 - 2}$ & $\mathbf{2 - 4}$ & $\mathbf{7}$ \\
\cline { 2 - 8 } & 3 & 4 & 12 & 24 & 25 & 22 & 10 \\
\hline Bagaço de cana-de-açúcar (BC) & 2 & 6 & 7 & 12 & 23 & 49 & 1 \\
Casca de amendoim (CA) & 4 & 32 & 49 & 11 & 2 & 2 & 0 \\
Areia (A) & 4 & 31 & 48 & 11 & 3 & 2 & 1 \\
$2 / 3 \mathrm{~A}+1 / 3 \mathrm{BC}$ & 2 & 27 & 48 & 12 & 5 & 6 & 0 \\
$2 / 3 \mathrm{~A}+1 / 3 \mathrm{CA}$ & 3 & 30 & 48 & 11 & 4 & 4 & 0 \\
$2 / 3 \mathrm{~A}+1 / 6 \mathrm{BC}+1 / 6 \mathrm{CA}$ & 3 & 29 & 49 & 12 & 3 & 3 & 1 \\
$1 / 2 \mathrm{~A}+1 / 2 \mathrm{BC}$ & 2 & 29 & 46 & 11 & 5 & 7 & 0 \\
$1 / 2 \mathrm{~A}+1 / 2 \mathrm{CA}$ & 4 & 26 & 42 & 13 & 6 & 8 & 1 \\
$1 / 3 \mathrm{~A}+1 / 3 \mathrm{BC}+1 / 3 \mathrm{CA}$ & & & & & &
\end{tabular}

Tabela 2. Teores de magnésio $(\mathrm{Mg})$, boro (B) e zinco $(\mathrm{Zn})$, aos 41 dias após o transplantio, nas folhas do tomateiro do grupo cereja, cultivar Sindy, cultivado em sete substratos, em casa de vegetação. Jaboticabal, FCAV-UNESP, 2004.

\begin{tabular}{lccc}
\hline Substratos $^{1}$ & $\left.\mathbf{M g} \mathbf{( g ~ \mathbf { ~ g } ^ { - 1 }}\right)$ & $\mathbf{B}\left(\mathbf{m g ~ k g}^{-1}\right)$ & $\left.\mathbf{Z n} \mathbf{~ m g ~ k g}^{-1}\right)$ \\
\hline $\mathrm{A}$ & $6,4 \mathrm{a}$ & $137 \mathrm{a}$ & $67 \mathrm{a}$ \\
$2 / 3 \mathrm{~A}+1 / 3 \mathrm{BC}$ & $4,8 \mathrm{~b}$ & $107 \mathrm{~b}$ & $44 \mathrm{bc}$ \\
$2 / 3 \mathrm{~A}+1 / 3 \mathrm{CA}$ & $5,8 \mathrm{ab}$ & $135 \mathrm{a}$ & $61 \mathrm{ab}$ \\
$2 / 3 \mathrm{~A}+1 / 6 \mathrm{BC}+1 / 6 \mathrm{CA}$ & $5,4 \mathrm{ab}$ & $118 \mathrm{ab}$ & $54 \mathrm{abc}$ \\
$1 / 2 \mathrm{~A}+1 / 2 \mathrm{BC}$ & $4,6 \mathrm{~b}$ & $118 \mathrm{ab}$ & $38 \mathrm{c}$ \\
$1 / 2 \mathrm{~A}+1 / 2 \mathrm{CA}$ & $5,1 \mathrm{ab}$ & $126 \mathrm{ab}$ & $57 \mathrm{abc}$ \\
$1 / 3 \mathrm{~A}+1 / 3 \mathrm{BC}+1 / 3 \mathrm{CA}$ & $4,5 \mathrm{~b}$ & $119 \mathrm{ab}$ & $44 \mathrm{bc}$ \\
\hline $\mathrm{CV}(\%)$ & 10,8 & 8,2 & 17,8 \\
\hline
\end{tabular}

${ }^{1} \mathrm{~A}=$ areia; $\mathrm{BC}=$ bagaço de cana-de-açúcar; $\mathrm{CA}=$ casca de amendoim;

Médias, seguidas da mesma letra na coluna, não diferem entre si pelo teste de Tukey a $5 \%$ de probabilidade.

volume de água, em $\mathrm{L}$ planta ${ }^{-1} \operatorname{dia}^{-1}$, foi igual a 0,780 durante os primeiros 15 dias após o transplantio (DAT), com freqüência de irrigação (FI) igual a quatro vezes ao dia; 0,975 do $16^{\circ}$ ao $30^{\circ} \mathrm{DAT}$, com FI $=5$ vezes ao dia; 1,300 do $31^{\circ}$ ao $45^{\circ} \mathrm{DAT}$, com FI $=5$ vezes ao dia; 1,560 do $46^{\circ}$ ao $60^{\circ} \mathrm{DAT}$, com $\mathrm{FI}=6$ vezes ao dia; 1,950 do $61^{\circ}$ ao $75^{\circ} \mathrm{DAT}$, com FI $=6$ vezes ao dia; 2,548 do $76^{\circ}$ ao $90^{\circ} \mathrm{DAT}$, com FI $=7$ vezes ao dia; 3,276 do $91^{\circ}$ ao $122^{\circ} \mathrm{DAT}$, com $\mathrm{FI}=7$ vezes ao dia. $\mathrm{A}$ irrigação foi realizada por gotejamento, utilizando-se um gotejador por vaso, com vazão de $13 \mathrm{~mL} \mathrm{~min}{ }^{-1}$.

A fertirrigação foi realizada constantemente, ou seja, os fertilizantes foram aplicados em todas as irrigações. Para o fornecimento de nutrientes, utilizou-se a solução nutritiva recomendada por Moraes (1997) para o cultivo do tomateiro em sistema NFT (Técnica do Fluxo Laminar de Nutrientes). Para o preparo de 1000 L de solução nutritiva, utilizou-se $285 \mathrm{~g} \quad \mathrm{de}$ monoamoniofosfato; $600 \mathrm{~g}$ de sulfato de magnésio; 1088 g de nitrato de cálcio; $423 \mathrm{~g}$ de sulfato de potássio; $340 \mathrm{~g}$ de cloreto de potássio; $3 \mathrm{~g}$ de sulfato de manganês; $0,45 \mathrm{~g}$ de sulfato de zinco; $2,94 \mathrm{~g}$ de ácido bórico; $10 \mathrm{~g}$ de sulfato de ferro; $0,41 \mathrm{~g}$ de sulfato de cobre e $0,02 \mathrm{~g}$ de molibdato de sódio. A quantidade total de cada nutriente aplicada ao longo do ciclo da cultura (122 dias), em g planta ${ }^{-1}$, foi de: 41 de nitrogênio; 12 de fósforo; 72 de potássio; 42 de cálcio; 12 de magnésio; 31 de enxofre; 0,10 de boro; 0,02 de cobre; 0,41 de ferro; 0,15 de manganês; 0,02 de zinco e 0,002 de molibdênio.

Para o controle de pragas e doenças, foram realizadas, conforme a necessidade, pulverizações com defensivos nas doses e épocas recomendadas pelo fabricante para a cultura do tomate.

A colheita iniciou-se aos 62 dias após o transplantio e teve duração de 60 dias. A colheita dos frutos foi realizada duas vezes por semana.

Realizou-se a amostragem das folhas no período de pleno florescimento da cultura, que se deu aos 41 dias após o transplantio, segundo método proposto por Malavolta et al. (1997). As amostras de folhas foram lavadas em água deionizada, colocadas para secar em estufa com circulação forçada de ar a $60^{\circ} \mathrm{C}$, até atingirem peso constante e, posteriormente, moídas para serem submetidas à análise química, segundo método descrito por Bataglia et al. (1983).

As variáveis avaliadas foram submetidas à análise de variância e as médias comparadas pelo teste de Tukey a 5\% de probabilidade.

\section{RESULTADOS E DISCUSSÃO}

Os teores foliares de magnésio $(\mathrm{Mg})$, boro (B) e zinco (Zn), aos 41 dias após o transplantio, foram diferentes nas plantas cultivadas nos substratos, embora as quantidades dos nutrientes fornecidos tenham sido as mesmas (Tabela 2). Assim, os substratos avaliados proporcionaram ao sistema radicular diferentes condições para a absorção de nutrientes.

Para os demais nutrientes, os teores foliares foram estatisticamente iguais e variaram dentro dos seguintes intervalos: $\mathrm{N}$ de 58,1 a $63,6 \mathrm{~g} \mathrm{~kg}^{-1} ; \mathrm{P}$ de 5,1 a $5,8 \mathrm{~g} \mathrm{~kg}^{-1} ; \mathrm{K}$ de 29,0 a 33,7 $\mathrm{g} \mathrm{kg}^{-1} ; \mathrm{Ca}$ de 16,0 a $18,0 \mathrm{~g} \mathrm{~kg}^{-1} ; \mathrm{S}$ de 10,5 a $11,6 \mathrm{~g} \mathrm{~kg}^{-1}$; $\mathrm{Cu}$ de 368 a $540 \mathrm{mg} \mathrm{kg}^{-1}$; Fe de 438 a $1975 \mathrm{mg} \mathrm{kg}^{-1}$; Mn de 162 a $500 \mathrm{mg} \mathrm{kg}^{-1}$.

De maneira geral, independentemente do substrato, os teores de nutrientes nas folhas do tomateiro, aos 41 dias após o transplantio, foram superiores aqueles considerados adequados por Malavolta et al. (1997) $(\mathrm{N}=30, \mathrm{P}=3,5$, $\mathrm{K}=40, \mathrm{Ca}=14-18, \mathrm{Mg}=4, \mathrm{~S}=3$, em $\mathrm{g} \mathrm{kg}^{-1}$; $\mathrm{B}=50-70, \mathrm{Cu}=10-15, \mathrm{Fe}=500-700$, $\mathrm{Mn}=250-400, \mathrm{Zn}=60-70$, em mg kg-1). Ainda que os próprios autores considerem esses valores indicações gerais, podendo as condições do meio radicular, do clima e da variedade influenciá-los, observou-se, nas condições deste experimento, teores foliares elevados. Essa constatação indica que o constante fornecimento da solução nutritiva com a concentração de nutrientes utilizada disponibilizou ao sistema radicular 
quantidade de nutrientes maior que a considerada adequada para a planta. Portanto verifica-se a necessidade de mais estudos a respeito da concentração da solução nutritiva fornecida, via fertirrigação, para o cultivo do tomateiro do grupo cereja, cultivar Sindy, em substratos.

Os valores da densidade (D), no final do cultivo, decresceram com a diminuição do volume de areia e o aumento de bagaço de cana-de-açúcar (BC) e/ ou casca de amendoim (CA) (Tabela 3 ). Pode-se observar que, naqueles substratos nos quais a proporção de areia foi a mesma, os valores de D foram estatisticamente iguais, independentemente do componente ser $\mathrm{BC}, \mathrm{CA}$ ou $\mathrm{BC}+$ CA. Assim, conforme o esperado, a areia foi o componente determinante no valor final da densidade dos diferentes substratos.

Observaram-se diferenças significativas para os valores do espaço de aeração e da água facilmente disponível dos substratos no final do cultivo (Tabela 3). Tais diferenças observadas foram influenciadas pela distribuição do tamanho das partículas (granulometria) dos substratos (Tabela 1), uma vez que esta afeta a distribuição do tamanho dos poros formados. Partículas com diâmetro menor que $0,25 \mathrm{~mm}$ formam poros em que a água neles armazenada, por estar mais fortemente retida, será de mais difícil disponibilidade às plantas; partículas com diâmetro entre 0,25 e $1,0 \mathrm{~mm}$ formam poros responsáveis pelo armazenamento de água facilmente disponível às plantas e partículas com diâmetro maior que 1,0 mm formam poros responsáveis pelo armazenamento de ar (Richards et al., 1986; Abad et al., 2004).

O menor valor do espaço de aeração (EA) foi observado no substrato composto por areia (Tabela 3), que apresentou predominância de partículas com diâmetro entre 0,125 e 0,50 mm (Tabela 1). A redução da proporção volumétrica de areia e conseqüente aumento de $\mathrm{BC}$ e/ou CA proporcionou aumento nos valores de EA (Tabela 3 ), devido ao aumento na porcentagem de partículas com diâmetro maior que 1,0 mm (Tabela 1). Comparando-se os substratos $1 / 2 \mathrm{~A}+1 / 2 \mathrm{BC}$ e $1 / 2 \mathrm{~A}+1 / 2$ $\mathrm{CA}$, observou-se que o substrato com

Tabela 3. Densidade (D), espaço de aeração (EA) e água facilmente disponível (AFD) dos substratos e produtividade (P) do tomateiro do grupo cereja, cultivar Sindy, cultivado em sete substratos, em casa de vegetação. Jaboticabal, FCAV-UNESP, 2004.

\begin{tabular}{|c|c|c|c|c|}
\hline Substratos $^{1}$ & $D\left(\mathrm{~kg} \mathrm{~m}^{-3}\right)$ & EA (\%) & AFD (\%) & $P\left(\mathrm{~kg} \mathrm{~m}^{-2}\right)$ \\
\hline $\bar{A}$ & $1604 \mathrm{a}$ & $2 d$ & $23 a$ & $8,9 a$ \\
\hline $2 / 3 A+1 / 3 B C$ & $1414 b$ & $3 d$ & $25 a$ & $10,7 \mathrm{a}$ \\
\hline $2 / 3 A+1 / 3 C A$ & $1379 b$ & $4 \mathrm{~d}$ & $23 a$ & $8,5 \mathrm{a}$ \\
\hline $2 / 3 A+1 / 6 B C+1 / 6 C A$ & $1353 b$ & $4 d$ & $25 a$ & 9,8 a \\
\hline $1 / 2 A+1 / 2 B C$ & $1142 \mathrm{c}$ & $9 \mathrm{c}$ & $24 a$ & $10,6 \mathrm{a}$ \\
\hline $1 / 2 A+1 / 2 C A$ & $1073 \mathrm{c}$ & $14 \mathrm{~b}$ & $19 b$ & $10,0 \mathrm{a}$ \\
\hline $1 / 3 A+1 / 3 B C+1 / 3 C A$ & $790 \mathrm{~d}$ & $21 \mathrm{a}$ & $14 \mathrm{c}$ & $9,8 \mathrm{a}$ \\
\hline CV (\%) & 4,2 & 23,0 & 10,1 & 11,5 \\
\hline
\end{tabular}

${ }^{1} \mathrm{~A}=$ areia; $\mathrm{BC}=$ bagaço de cana-de-açúcar; $\mathrm{CA}=$ casca de amendoim;

Médias, seguidas da mesma letra na coluna, não diferem entre si pelo teste de Tukey a $5 \%$ de probabilidade.

$\mathrm{BC}$ apresentou menor valor de $\mathrm{EA}(\mathrm{Ta}-$ bela 3), devido à menor porcentagem de partículas com diâmetro maior que 1,00 mm do que o substrato com CA (Tabela 1). O componente $\mathrm{BC}$ apresentou intervalo de tamanho de partículas mais amplo (diâmetro maior que $0,25 \mathrm{~mm}$ ), quando comparado ao componente CA, com partículas entre 1,00 e 4,00 mm (Tabela 1). Portanto, a utilização do componente CA contribuiu para aumentar o volume do espaço de aeração dos substratos.

Os menores valores de água facilmente disponível (AFD) foram observados nos substratos com $1 / 2 \mathrm{~A}+1 / 2$ CA e 1/3 A + 1/3 BC + 1/3 CA (Tabela $3)$, que apresentaram as menores porcentagens de partículas com diâmetro entre 0,25 e 1,0 $\mathrm{mm}$ (Tabela 1).

Embora os substratos avaliados tenham apresentado diferentes valores de densidade (D), espaço de aeração (EA) e água facilmente disponível (AFD), a produtividade do tomateiro cultivado nos diferentes substratos foi estatisticamente igual, variando entre 8,5 e 10,7 $\mathrm{kg} \mathrm{m}^{-2}$ (Tabela 3). Portanto, nas condições deste experimento, substratos com valores de D entre 790 e $1604 \mathrm{~kg} \mathrm{~m}^{-3}$, EA entre 2 e $21 \%$ e AFD entre 14 e $25 \%$ foram adequados para o cultivo do tomateiro do grupo cereja, cultivar Sindy, em casa de vegetação.

Outros autores conduziram experimentos com tomateiro na região de Jaboticabal. Pádua et al. (2002) observaram produtividade entre 6 e $8 \mathrm{~kg} \mathrm{~m}^{-2}$ para as cultivares de tomate cereja Cheri e Sindy cultivadas em substrato comercial; Gusmão et al. (2004) obtiveram produtividade entre 5 e $6 \mathrm{~kg} \mathrm{~m}^{-2}$ para as cul- tivares de minitomate Mascot, Gisela, Cheri e Sweet Million cultivadas em areia. Nota-se, portanto, que as produtividades obtidas no presente experimento foram superiores àquelas apresentadas por Pádua et al. (2002) e Gusmão et al. (2004). Este fato indica que o bagaço de cana-de-açúcar e a casca de amendoim podem ser utilizados na composição de substratos à base de areia, para o cultivo do tomateiro do grupo cereja, cultivar Sindy, em casa de vegetação.

\section{AGRADECIMENTOS}

Ao CNPq, pela concessão da bolsa de doutorado.

\section{LITERATURA CITADA}

ABAD MB; NOGUERA PM; CARRIÓN CB. 2004. Los sustratos en los cultivos sin suelo. In: URRESTARAZU MG. (Ed.). Tratado de cultivo sin suelo. Madrid: Mundi-Prensa, p. 113-158. ANDRIOLO JL; DUARTE TS; LUDKE L; SKREBSKY EC. 1999. Caracterização e avaliação de substratos para o cultivo do tomateiro fora do solo. Horticultura Brasileira, 17: 215-219.

ANDRIOLO JL. 1996. O cultivo de plantas com fertirrigação. Santa Maria: UFSM, 47 p.

BACKES MA. 1988. Composto de lixo urbano como substrato para plantas ornamentais. $80 \mathrm{f}$. Dissertação, Faculdade de Agronomia, Universidade Federal do Rio Grande do Sul, Porto Alegre. BATAGLIA OC; FURLANI AMC; TEIXEIRA JPF; FURLANI PR; GALLO JR. 1983. Métodos de análise química de plantas. Campinas: Instituto Agronômico, 48 p. (Boletim Técnico, 78).

BIASI LA; BILIA DAC; SÃO JOSÉ AR; FORNASIERI JL; MINAMI K. 1995. Efeito de misturas de turfa e bagaço-de-cana sobre a produção de mudas de maracujá e tomate. Scientia Agricola, 52: 239-243.

BURÉS S. 1997. Sustratos. Madrid: Agrotécnicas, 342 p. 
CARRIJO OA; VIDAL MC; REIS NVB; SOUZA RB; MAKISHIMA N. 2004. Produtividade do tomateiro em diferentes substratos e modelos de casas de vegetação. Horticultura Brasileira, 22: 05-09.

De BOODT M; VERDONCK O. 1972. The physical properties of the substrates in horticulture. Acta Horticulturae, 26: 37-44.

De BOODT M; VERDONCK O; CAPPAERT I. 1974. Method for measuring the waterrelease curve of organic substrates. Acta Horticulturae, 37 2054-2062.

FERNANDES C; ARAÚJO JAC; CORÁ JE. 2002. Impacto de quatro substratos e parcelamento da fertirrigação na produção de tomate sob cultivo protegido. Horticultura Brasileira, 20: 559-563.

GUSMÃO MTA; GUSMÃO SAL; ARAÚJO

JAC. 2004. Produtividade de minitomate cultivado em ambiente protegido e em diferentes substratos. Horticultura Brasileira, 22: 2, Suplemento 2. CD-ROM. Trabalho apresentado no $44^{\circ}$ Congresso Brasileiro de Olericultura, 2004.
KÄMPF AN; JUNG M. 1991. The use of carbonized rice hulles as an horticultural substrate. Acta Horticulturae, 294: 271-283.

MALAVOLTA E; VITTI GC; OLIVEIRA SA. 1997. Avaliação do estado nutricional das plantas: princípios e aplicações. 2.ed. Piracicaba: POTAFOS, $319 \mathrm{p}$

MORAES CAG. 1997. Hidroponia: Como cultivar tomates em sistema NFT (Técnica do fluxo laminar de nutrientes). Jundiaí: DISQ Editora, 141p.

NOGUERA P; ABAD M; PUCHADES R; NOGUERA V; MAQUIEIRA A; MARTÍNEZ J. 1997. Physical and chemical properties of coir waste and their relation to plant growth. Acta Horticulturae, 450: 365-373.

PÁDUA JG; GUSMÃO SAL; GUSMÃO MTA; BRAZ LT. 2002. Densidade de plantio e produção de duas cultivares de tomateiro tipo cereja, cultivadas em substrato, sob condições protegidas. Horticultura Brasileira, 20: 2, Suplemento 2. CD-ROM. Trabalho apresentado no $42^{\circ}$ Congresso Brasileiro de Olericultura, 2002.
REIS M; INÁCIO H; ROSA A; CAÇO J; MONTEIRO A. 2001. Grape marc compost as an alternative growing media for greenhouse tomato. Acta Horticulturae, 554: 75-81.

RICHARDS D; LANE M; BEARDSELL DV. 1986. The influence of particle-size distribution in pinebark:sand:brown coal potting mixes on water supply aeration and plant growth. Scientia Horticulturae, 29: 1-14.

SONNEVELD C; ENDE J; DIJK PA. 1974. Analysis of growing media by means of a $1: 1,5$ volume extract. Communications in Soil Science and Plant Analysis, 5: 183-202.

URRESTARAZU M; SALAS MC; RODRÍGUEZ R; ELORRIETA MA; MORENO J. 2000. Evaluación agronómica del uso del compost de residuos hortícolas como sustrato alternativo en cultivo sin suelo en tomate. Actas de Horticultura, 32: 327-332.

VERDONCK O. 1983. Reviewing and evaluation of new materials used as substrates. Acta Horticulturae, 150: 467-473. 